\title{
Distribution of Visual Attention within a Cued Area: Evidence Based on Temporal Order Judgments
}

\author{
Distribuição da Atenção Visual dentro de Áreas Indicadas: Evidência \\ Baseada em Julgamentos de Ordem Temporal
}

\author{
Mikael Cavallet ${ }^{a}$, Cesar Alexis Galera ${ }^{*}, a$, Michael W. von Grünau ${ }^{b}, \&$ Afroditi Panagopoulos ${ }^{b}$ \\ ${ }^{a}$ Universidade de São Paulo, Ribeirão Preto, Brasil \\ $\&{ }^{b}$ Departament of Psychology, Concordia University, Canada
}

\begin{abstract}
Three experiments were performed to investigate the distribution of attention across the visual field and the possibility of attentional resources to be more concentrated inside an abrupt onset frame (cue). The participants performed a temporal order judgment task of two letters presented in sequence; one letter presented inside and the other outside the frame. The results showed that the information presented inside the frame had its perceptual latency shortened in relation to the information presented outside the frame in experimental conditions where the frame orientation, the distance between the two letters and the cue onset time were manipulated. The advantage of the information presented inside the frame was attributed to the displacement of attention to the area delimited by the frame. The results contribute to the understanding of visual perception, showing that attentional resources may be redistributed inside the borders of a geometric figure.

Keywords: Visual attention; Cue; Border; Temporal Order Judgment Task.

\section{Resumo}

Três experimentos foram realizados para investigar a distribuição da atenção pelo campo visual e a possibilidade dos recursos de atenção serem mais concentrados no interior de uma moldura (dica) de início abrupto. Os participantes realizaram uma tarefa de julgamento de ordem temporal de duas letras apresentadas em seqüência; uma letra apresentada dentro e a outra fora da moldura. Os resultados mostraram que a informação apresentada dentro da moldura teve a sua latência perceptual encurtada em relação à informação apresentada fora da moldura em condições experimentais onde a orientação da moldura, a distância entre as duas letras e o tempo de exposição da moldura foram manipulados. Esta vantagem para a informação apresentada dentro da moldura foi atribuída ao deslocamento da atenção para a área delimitada pela moldura. Os resultados contribuem para o entendimento da percepção visual, mostrando que recursos de atenção podem ser redistribuídos dentro das bordas de uma figura geométrica.

Palavras-chave: Atenção visual; Dica; Borda; Tarefa de Julgamento de Ordem Temporal.
\end{abstract}

Several studies have shown that the displacement of spatial attention to a specific visual stimulus can influence the processing of attended and not attended events: in general, faster reaction times and greater accuracy are observed with attended than with not attended stimuli, even when the gaze is not directed to the area where the stimuli appear (covert attention). A simple way to get these benefits is the employment of peripheral or central cues, which are presented before the relevant stimulus (target)

"Address: Universidade de São Paulo, Faculdade de Filosofia, Ciências e Letras, Av. Bandeirantes, 3900 Monte Alegre, Ribeirão Preto, SP, Brasil, CEP 14040901. E-mail: cesar_galera@ffclrp.usp.br

Acknowledgement: This study was supported by $\mathrm{Co}-$ ordenação de Aperfeiçoamento de Pessoal de Nível Superior (CAPES) for the task and indicate, with a certain probability, the location where this target can appear. These two kinds of cues produce different patterns of results and are related to two different ways to engage visual attention in a specific task. Peripheral cues, like a stimulus presented abruptly in the periphery of the visual field, are assumed to orient attention automatically, while central cues orient attention in a controlled way (H. J. Müller \& Rabbitt, 1989). The facilitation observed when peripheral cues are used has a peak in performance at around $100 \mathrm{~ms}$ after the onset of the cue, followed by a decrease after 300 $\mathrm{ms}$, indicating that different phases of allocation of attention could exist with an automatic transitory initial phase, while later it may be followed by a voluntary component that can be extended for a longer period (Cheal \& Lyon, 1991; H. J. Müller \& Rabbitt, 1989; Nakayama $\&$ Mackeben, 1989). The initial advantage observed 
around $100 \mathrm{~ms}$ after cue onset can also be followed by an inhibition of the target reaction time on the cued side, in comparison to the uncued side at about $300 \mathrm{~ms}$ (Posner \& Cohen, 1984). This inhibition also known as inhibition of return, might promote the sampling of areas not yet visited (Posner \& Cohen, 1984). On the other hand, central cues, like an arrow presented at the center of the screen pointing in the direction where the participant should attend, produce a gradual increase in facilitation after longer intervals (greater than $300 \mathrm{~ms}$ ) and are more durable before fading out.

The employment of cues to investigate the distribution of attention has given important information about the attentional mechanisms and characteristics. Posner, Snyder and Davidson (1980) found that visual attention cannot be allocated freely to several positions in space, but appears to have a central focus that can be allocated just to a single position in the scene. Corroborating the finding of Posner et al. (1980), more recent evidence from physiological data shows that information covered by the attentional focus receives an advantage in processing, while the information not included in it is suppressed or only partially processed (N. G. Müller \& Kleinschmidt, 2004). There is also evidence that the focus can be variable in size, being able to be adjusted to small and large areas (Castiello \& Umiltà, 1990; Eriksen \& St. James, 1986; Eriksen \& Yeh, 1985; LaBerge, 1983). Furthermore, the attentional focus seems to be distributed in a gradient way, so that the processing advantage of a target presented outside, but close to the cued area, decreases gradually with distance, to finally reach the effect of a target presented more distant from the center of the focus (Laberge \& Brown, 1989; Shulman, Wilson, \& Sheehy, 1985).

Although the idea of the focus suggests that the attentional focus might occupy a simple and single continuous region of the visual field (Eriksen \& St. James, 1986; Eriksen \& Yeh, 1985; Posner et al., 1980), it is not clear how the processing occurs in more complex spatial configurations. Behavioral and physiological investigations provide evidence for the idea that the focus of attention is flexible in relation to the form of the cued area, being modulated by different shapes (Egly \& Homa, 1984; Juola, Bouwhuis, Cooper, \& Warner, 1991; M. M. Müller \& Hübner, 2002). Other studies indicate that after the initial displacement of attention to a frame, the focus can be adjusted to the limits of the frame, being influenced by its size and shape (Benso, Turatto, Mascetti, \& Umiltà, 1998; Maringelli \& Umiltà, 1998). In the same way, more recent studies using geometric figures as cues suggest the automatic (Galera, von Grünau, \& Panagopoulos, 2005) and controlled (Panagopoulos, von Grünau, \& Galera, 2004) distribution of visual attention within the area delimited by a frame of rectangular shape.

On the other hand, this process of distribution and adjustment of the focus of attention to the borders of a geometric figure does not seem to be so clear and simple. Results of Experiment 2 of Castiello and Umiltà (1990) leave open the possibility of a gradual distribution of attention near the borders of a delimited area. There are also results partially contrary to the idea of the adjustment of attention to circular shapes, which raises doubts about the distribution of visual attention at the borders of areas delimited by cues (Eimer, 2000; Juola, Crouch, \& Cocklin, 1987).

The present study investigated this topic exploring the distribution of visual attention when a rectangular framelike cue was presented abruptly in the visual field. Although the frame was not mentioned to the participants and did not predict the location of the first target used in the task, it might be considered as a predictive cue, because it indicated the direction or region where the targets could appear. The participants performed a temporal order judgment (TOJ) task, and the principal idea was that the resources of attention should be more concentrated inside the frame, spreading along the whole frame, than outside it. The experiments were based on evidence that an abrupt onset automatically reallocates visual attention (Remington, Johnston, \& Yantis 1992; Turatto et al., 2000; Yantis \& Jonides, 1990, 1996), as well as, on evidence that the resources of attention can be reallocated to an area delimited by a specific shape (Benso et al., 1998; Egly \& Homa, 1984; Galera et al., 2005; Juola et al., 1991; M. M. Müller \& Hübner, 2002; Panagopoulos et al., 2004).

The TOJ task was chosen to investigate this topic because it might provide an alternative measure of the concentration of resources that differs from the measures more often obtained by using RT tasks. The use of the TOJ task can provide results that are less influenced by motor components, because the emphasis is on the accuracy and not on the speed of the responses (Cole, Gellatly, \& Blurton, 2001). Also, the TOJ task allows for the comparison between the judgment of a target presented at a location delimited by a cue and at a location not cued at the same time (Scharlau, 2004). This comparison seems useful because it can provide important information about the performance and processing at different areas that theoretically receive different amounts of attention. This kind of paradigm has been used in different studies to investigate visual attention, and the results demonstrate that the perceptual latency is shorter for attended than for not attended stimuli, revealing the prior entry effect (Abrams \& Law, 2000; Hikosaka, Miyauchi, \& Shimojo, 1993; McDonald, Teder-Sälejärvi, Di Russo, \& Hillyard, 2005; Scharlau, 2004; Shore, Spence \& Klein, 2001; Stelmach \& Herdman, 1991; Sternberg \& Knoll, 1973; Vibell, Klinge, Zampini, Spence, \& Nobre, 2007). Therefore, the results of this study can contribute to the understanding of the processing of information presented inside and outside the borders of a figure with an abrupt onset. 


\section{Experiments}

\section{Purpose}

Experiment 1 was designed to investigate if the abrupt onset of a rectangular frame presented for $100 \mathrm{~ms}$ before the presentation of two letters interferes in the perception of their temporal order. According to the idea of prior entry, the automatic allocation of attention to a specific stimulus triggered by the presentation of a peripheral cue should produce a great advantage in processing around $100 \mathrm{~ms}$ for this attended stimulus in relation to a not attended stimulus (Shore et al., 2001; Stelmach \& Herdman, 1991). As a result, if two visual stimuli are presented simultaneously, the attended one has a shorter latency and more chance to be judged as being presented first than the not attended one. Thus, if two letters are presented in sequence with a variable interval between them, at certain interval, the letter presented inside the frame will be perceived as presented first in relation to the letter presented outside even though the letter presented inside has been shown after the letter presented outside. Experiment 1 was designed to explore this advantage.

Experiment 2 followed the same logic as Experiment 1 , and it was designed to investigate the influence of distance on the perception of temporal order, manipulating the spatial distance between the letter presented inside and the letter presented outside of the frame. The manipulation of the distance can provide evidence of the distribution of attention inside and near the area delimitated by the frame according to studies that have shown that the concentration of attention seems to diminish gradually from the initially attended area to more distant areas in experiments of reaction time (LaBerge \& Brown, 1989; Shulman et al., 1985) and TOJ tasks (Scharlau, 2004). The manipulation of the distance between the two letters might give important information about the distribution of attention since the distance should affect the perception of temporal order. Thereby, if the abrupt onset frame captures attention in an automatic way, the processing of the letter presented outside the frame will be less efficient as the distance between letter and frame increases because fewer resources would be available at more distant positions from the initial peak of activation of attention (LaBerge \& Brown, 1989; Shulman et al., 1985). Thus the distance between the letters was varied using the same TOJ task. The rectangle used in the first experiment was replaced by a square, and the letters were presented in different hemifields but arranged so that they had the same distance from the fixation stimulus. These modifications permitted better control of the distance between the letters and the geometric center of the square, and had the same distance from fixation, allowing control of eccentricity.

Considering that attention is attracted to the delimited area used in Experiments 1 and 2, giving an advantage for information presented inside the frame, this advantage should also have a temporal dynamic similar to that found in experiments with peripheral cues, where a peak in performance is found at short cue-target intervals of time and a reduced (Cheal \& Lyon, 1991; H. J. Müller \& Rabbitt, 1989; Nakayama \& Mackeben, 1989) or inhibited advantage at longer intervals (Posner \& Cohen, 1984). Thus, in Experiment 3 the interval between the frame and the first letter was manipulated to investigate the temporal dynamic of this advantage. In this way, if the frame was presented before the first letter during a variable time starting at short values such as $60 \mathrm{~ms}$ to long values like $410 \mathrm{~ms}$, the advantage of the letter presented inside of the frame should reach a maximum value at about 100 $\mathrm{ms}$ after the start of the frame presentation and a decrease or inhibition at about $300 \mathrm{~ms}$ producing a characteristic distribution of automatic attention during the presentation of the frame, as demonstrated in the experiments with cues. The decrease or inhibition in performance can be verified by the analyses of the cost in processing of the letter presented outside the frame revealing a decrease (Nakayama \& Mackeben, 1989) or an inhibition in performance (Posner \& Cohen, 1984).

\section{General Method}

In all experiments, a frame and two letters were presented in sequence; one letter inside and the other outside of the frame. The order of presentation of the two letters was manipulated, and the participant judged which letter appeared first. The advantage of the letter presented inside in relation to the letter presented outside of the frame was obtained through half of the difference between the point of subjective simultaneity (PSS) of the condition inside and the condition outside of the frame (Shore et al., 2001). This advantage was named perceptual facilitation (PF) and PSS represents the interval of time between the presentations of the two stimuli to be judged, in which the observer is more uncertain about the order of the stimuli presented in each condition. Thus, if the presentation of the frame induces the effect of prior entry, the PSS of the letter presented first inside should be different from the PSS of the letter presented first outside, respectively, revealing the gain and the cost of the positions of the letters, as well as the PF produced by the frame.

\section{Participants}

A total of forty students from the University of São Paulo - USP and an author (MC), participated in this study. Two participants took part in all experiments, one participant participated in Experiments 1 and 2 and four participated in Experiments 2 and 3. Experiments were carried out in sequence, with an interval of more than one month between them. Eight participants (4 female), average of 26 years $(S D=3)$ completed Experiment 1 , seventeen (11 female), average of 24 years $(S D=4)$ completed Experiment 2 and fifteen ( 5 female), average 
of 24 years $(\mathrm{SD}=5)$ completed Experiment 3, with a duration of approximately 17,30 and 45 minutes, respectively. All participants except the author did not have prior knowledge of the purpose of the study and all participants had normal or corrected-to-normal visual acuity. Experiments where performed in accordance with the ethical standards and all participants gave informed consent (process number: 180/2005- 2005.1.438.59.2).

\section{Apparatus and Stimuli}

The three experiments were generated and executed by E’Prime ${ }^{\circ} 1.1$ (Psychology Software Tools, Inc.; available at http://www.pstnet.com), and controlled by a Pentium IIIPC. The stimuli, letters "F" and "J" $\left(0.3^{\circ} \mathrm{x} 0.4^{\circ}\right.$ of visual angle and $0.1^{\circ}$ of thickness) and a frame (rectangle of $8.0^{\circ} \times 1.4^{\circ}$ or a square with sides of $1.8^{\circ}$ of visual angle, both with $0.1^{\circ}$ of thickness) were presented in black $(0.9$ $\left.\mathrm{cd} / \mathrm{m}^{2}\right)$ on a white background $\left(70 \mathrm{~cd} / \mathrm{m}^{2}\right)$ on a 17 -in monitor, with a resolution of $800 \times 600$ pixels and refresh rate of $100 \mathrm{~Hz}$. The rectangle was used in Experiments 1 and 3. It was presented in the vertical orientation to the left or right of fixation, or in the horizontal orientation above or below the fixation stimulus $\left(0.3^{\circ} \times 0.3^{\circ}\right.$ of visual angle). The distance between fixation and the geometric center of the rectangle was $1.1^{\circ}$ and the distance between the letters "F" and "J" was $2.2^{\circ}$ of visual angle. The square was used in Experiment 2, and its geometric center, as well as those of the two letters, were presented at the same distance from fixation forming an imaginary circle with a radius of $4.1^{\circ}$ of visual angle. Two distances between "F" and "J" were used: $1.9^{\circ}$ and $5.7^{\circ}$ of visual angle. The distance from the screen to the participant's eye was set at $58 \mathrm{~cm}$.

\section{Design}

In Experiment 1, when the frame was presented abruptly in the horizontal orientation above fixation (Figure 1A), the first letter (F or J) could be presented inside the frame, and above fixation with vertical eccentricity of $+1.1^{\circ}$ of visual angle, and the second letter outside of the frame, above or below fixation with vertical eccentricity of $+3.2^{\circ}$ or $-1.1^{\circ}$, respectively. The first letter could also be presented outside of the frame with the same eccentricities described for the position inside of the frame. The distances were equivalent when the frame was presented in the horizontal orientation below fixation and when it was presented abruptly in the vertical orientation to the left or the right of fixation. Both letters were never presented together inside or outside of the frame and they were always presented at adjacent positions to each other. In Experiment 1 there were 32 possible positions for presentation of the two letters (16 positions inside being 8 for vertical and 8 for horizontal orientation of the frame and 16 outside of the frame). In Experiment 3, we used the same design as in Experiment 1 with just one exception: the letter presented inside and the letter presented outside of the frame were always shown at the same eccentricity (Figure 1B). In Experiment 2, the square and the two letters were presented in two of 12 fixed locations around fixation, always in the opposite hemifield but never in the diagonal opposite hemifield (Figure 1C and D). In all experiments the letter " $\mathrm{F}$ " was presented first in $50 \%$ of the trials $(25 \%$ inside, $25 \%$ outside of the frame) and in the other $50 \%$ of the trials "J" was presented first ( $25 \%$ inside, $25 \%$ outside of the frame).

\section{Procedure}

The participants judged which of the two letters presented in sequence appeared first in each trial pressing with the index finger of each hand the corresponding letter on the computer keyboard. They were instructed to respond as accurately as possible. They were also informed to maintain the gaze on the fixation stimulus during the trials.

In all experiments each trial started with the presentation of the trial number for $500 \mathrm{~ms}$ to inform the participant about his/her progress in the block. Then the trial number was replaced by the fixation stimulus and after $300 \mathrm{~ms}$ the frame was presented abruptly. Both fixation and frame stayed on the screen until the end of the trial. The first letter was presented after a frame onset time of $100 \mathrm{~ms}$ and the second letter in sequence after a variable interval $(20,30,60,100$ or $200 \mathrm{~ms})$. After the second letter, all stimuli remained on the screen for $250 \mathrm{~ms}$ and after the participant's answer; the screen remained white for another $200 \mathrm{~ms}$. A new trial was then initiated. In Experiment 1, the frame orientation (horizontal or vertical), the first letter presented (F or J), the location of the first letter (inside or outside of the frame) and the variable interval $(20,30,60,100$ and $200 \mathrm{~ms})$ changed randomly in an experimental session of two blocks with 240 testtrials with twelve repetitions for each experimental treatment $(2 \times 2 \times 2 \times 5)$. In Experiment 2 there were three changes in the sequence of events: (a) In each trial, the participant pressed the space key to begin the trial; (b) after the participant's response, the fixation point changed to blue, signaling the end of the trial; and (c) the participants were instructed to take a break to rest when the fixation point was blue. All experimental treatments including the distance between the two letters $\left(1.9^{\circ}\right.$ or $5.7^{\circ}$ of visual angle) were randomized in 640 test-trials with sixteen repetitions for each factor $(2 \times 2 \times 2 \times 5)$. The sequence of events for Experiment 3 was the same as the sequence for Experiment 2 with one exception: the frame onset time was $60,110,210$ and $410 \mathrm{~ms}$ before the presentation of the first letter. All experimental treatments and the variable interval $(60,110,210$ and $410 \mathrm{~ms})$ were randomized in 640 test-trials with eight repetitions for each factor. Participants performed practice trials before the beginning of the blocks of test-trials in all experiments. They were also given an interval to rest between the blocks of test-trials. 
A

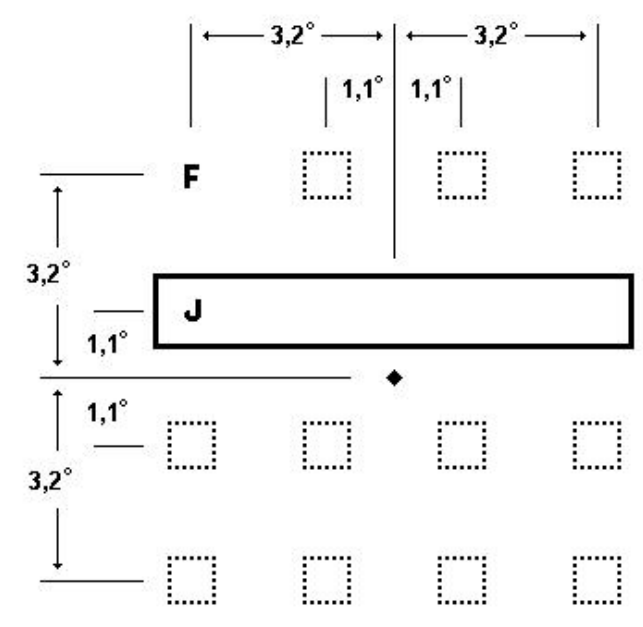

C

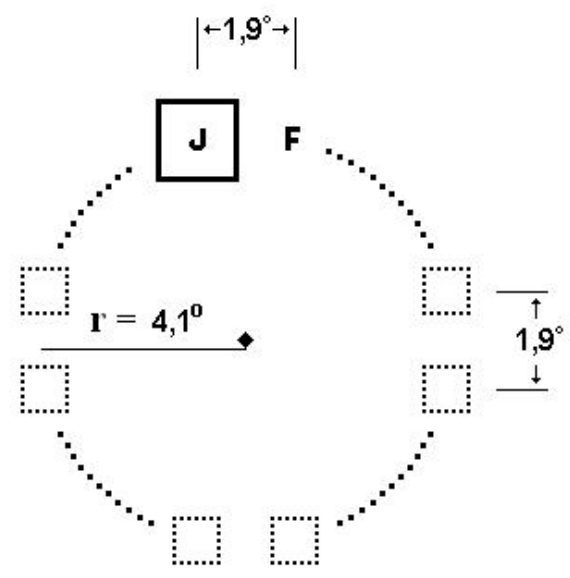

B

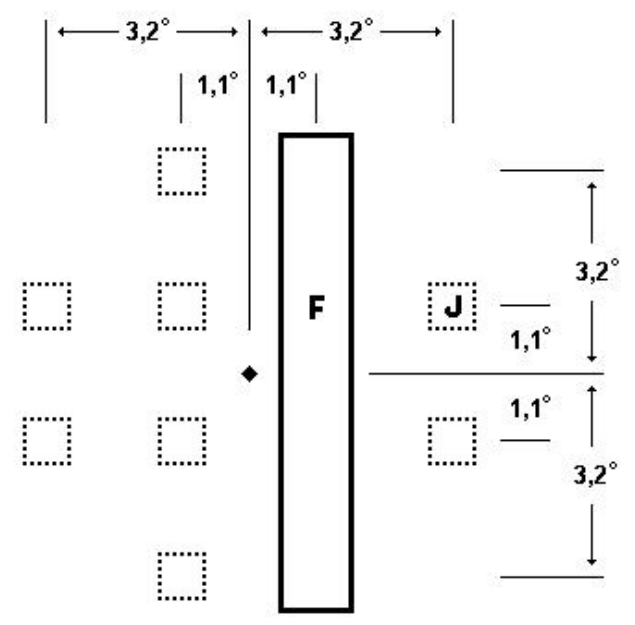

D

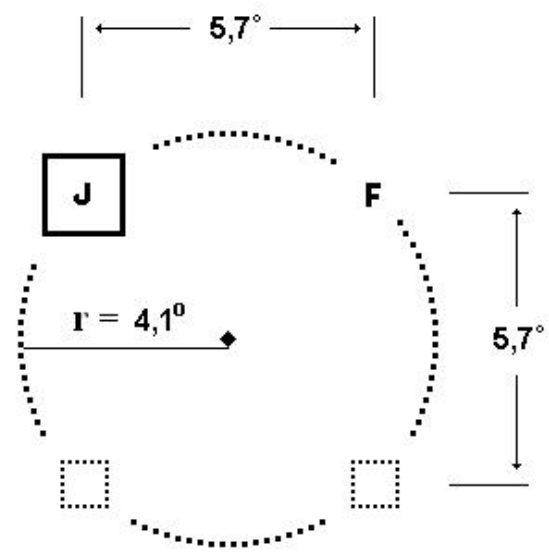

Figure 1. The letters (F or J) and the frame (rectangle or square) show examples of the distance and the positions in which the letters could be presented inside and outside of the frame in the three experiments. The stippled squares represent the possible locations where the two letters could appear. In Experiments 1 (A) and 3 (B) the frame could appear in the horizontal or vertical orientations, respectively above or below, right or left of the fixation stimulus. The letters were always presented one inside and the other outside and always at adjacent positions to each other (i.e. next closest parallel position). In Experiment 2 the letters were presented at $1.9^{\circ}(\mathrm{C})$ or at $5.7^{\circ}(\mathrm{D})$ of visual angle of each other. The geometric centers of the frame and the letters were always presented at $4.1^{\circ}$ of the fixation stimulus.

\section{Analysis}

The reaction time and the number of responses " $F$ " and "J" were recorded. Practice trials and abnormally slow responses (established by a skew, Schneider \& Bavelier, 2003) were discarded to avoid outlier responses. The PSS was obtained in two stages. Initially, the percentage of answers "F first" was calculated for each variable interval between the letters for each experimental condition. In sequence the distribution of the empiric values was fitted to the sigmoid Boltzmann function expressed by $Y=(A 1$ $-A 2) /\left(1+e^{\exp ((x-x)) /(x))+A 2}\right)$, where $Y$ is the judgement of "F" first in relation to the temporal interval $(x)$ between the two letters. $A 1$ is the initial $Y$ and A2 is the final $Y$. The midpoint $(x 0)$ of the Boltzmann function was used as PSS (50\% of responses), and $d x$ was the width of the function taken as a measure of accuracy of the perception of the temporal order.

\section{Results and Discussion of Experiment 1}

An average of $3.7 \%$ of trials was discarded due to very slow responses. The functions of conditions inside and outside of the frame of horizontal and vertical orientation 
can be seen in Figure 2A, where the negative values for the variable interval indicate that the letter " $\mathrm{J}$ " appeared first and the positive values that the letter "F" appeared first. The vertical arrows show the PSSs that correspond to $50 \%$ of responses " $\mathrm{F}$ first" in each function. An analysis of variance for repeated measures (ANOVA, $p<.05$ ) conducted on PSSs of each position of the first letter (inside or outside) and the frame orientation (horizontal or vertical) revealed a main effect of position (inside -43 $\mathrm{ms}$, outside $+35 \mathrm{~ms}, F(1.7)=70.98, p<.001)$. This means that there was a change in the perception of temporal order according to the position of the letter. The letter presented inside of the frame had an advantage in relation to the letter presented outside producing the significant displacement to the left (conditions inside) or to the right (conditions outside) of the fits. This result is also verified in the $\mathrm{PF}$ of $39 \mathrm{~ms}$ for information presented inside the frame. The effect can be attributed to the greater concentration of attention inside the frame. According to the prior entry hypothesis, the displacement of attention for a specific stimulus shortens the perceptual latency of this stimulus in relation to a stimulus that does not receive attention (Hikosaka et al., 1993; Mcdonald et al., 2005; Shore et al., 2001; Stelmach \& Herdman, 1991; Vibell et al., 2007).

The analysis also revealed that the frame orientation was not statistically significant (horizontal $-6 \mathrm{~ms}$, vertical -2 ms, $F(1.7)=3.68 ; p=.09$ ) and that there was a statistically significant interaction between frame orientation and the position of the first letter $(F(1.7)=6.66 ; p=$ $.04)$. Paired comparisons (Newman-Keuls post hoc test, $p<.05)$ showed that the PSS changed more when the first letter appeared inside the horizontal frame $(-51 \mathrm{~ms})$ than inside the vertical one $(-36 \mathrm{~ms})$. This difference did not occur when the first letter appeared outside the frame ( $38 \mathrm{~ms}$ and $32 \mathrm{~ms}$, respectively). The same pattern of results was found for the PF. A $t$ test $(p<.05)$ performed on the PFs of each frame orientation (horizontal or vertical) revealed larger PF for the frame presented in the horizontal $(44 \mathrm{~ms})$ than in the vertical orientation $(34 \mathrm{~ms}$, $t(7)=2.581 ; p=.04)$. These findings are similar to results of studies that have used RT tasks and have suggested different distributions of attention between the two meridians with greater concentration of attention on the horizontal than on the vertical meridian (Altpeter, Mackeben, \& Treuzettel-Klosinski, 2000; Galera et al., 2005; Panagopoulos et al. 2004). However, results manipulating visual restrictions and the displacement of attention indicate that such differences can be caused by visual factors, independent of the distribution of attention (Carrasco, Talgar, \& Cameron, 2001). Therefore, we can just suppose that the frame worked as a reference for a scale of distribution of visual attention, modulating the processing of the information presented inside of the frame.

However, considering the locations where the frame and the letters were presented in relation to the vertical and horizontal meridians, someone could ask if differen- ces in performance occurred between conditions in which the frame and the letter with position outside were presented in the same hemifield or in different hemifields, revealing an effect called "meridian effect". This effect was demonstrated by Rizzolatti, Riggio, Dascola and Umiltà (1987) in experiments based on the "Posner paradigm", where they found a cost of about $21 \mathrm{~ms}$ in reaction time for targets presented in an uncued location of the opposite hemifield of the cue compared to targets presented in an uncued location of the same hemifield, both at the same distance from the cue. The results revealed that when attention had to move from one hemifield to another, regardless of which meridian had to be crossed, there was a cost. Because the focus of attention should spend the same amount of time to move among different equidistant locations, this cost cannot be explained by the shift of attention between these locations presented in different hemifields. To explain this effect, Rizzolatti et al. (1987) elaborated the "premotor theory of attention", which claims that motor activity and attention are controlled by common mechanisms (see also Craighero \& Rizzolatti, 2005). They reported that when a cue is presented, a movement of the eyes is programmed to reach the target that might appear there, irrespective of whether the movement will be executed or not. If the target appears in a non-expected location, as the hemifield opposite to the attended one, the mechanism needs to reprogram the direction of the movement consuming more time than when only the distance needs to be reprogrammed (non-expected location within the attended hemifield). In this way, the same comparison between same and opposite hemifields for the letter presented outside of the frame was performed to verify if the PSSs reveal the meridian effect. The PSSs were reorganized and analyzed one more time, considering the position of the letter (inside or outside of the frame) and the hemifield of the letter presented outside (same or opposite to the frame hemifield) for horizontal and vertical meridians. The two way ANOVA conducted on the PSSs revealed a main effect for position $(-42 \mathrm{~ms}$ inside, $+30 \mathrm{~ms}$ outside, $F(1.7)=71.33 ; p<.01)$ and a statistically significant interaction between position and hemifield $(F(1.7)=14.93$; $p<.01$ ). Paired comparisons (Newman-Keuls post hoc test, $p<.05$ ) showed that the PSSs changed more when the letters were presented first outside in the opposite hemifield ( $+43 \mathrm{~ms}$ ) than in the same hemifield in which the frame was presented $(+17 \mathrm{~ms})$. This difference did not occur when the letter was presented first inside the frame (-34 ms same, $-48 \mathrm{~ms}$ opposite). Moreover, the difference between inside and outside was maintained for PSSs in the same and opposite hemifields.

These results showed a clear meridian effect in a TOJ paradigm. Moreover, the cost between the same and opposite hemifield, calculated by the difference between the PSSs of the two conditions was $27 \mathrm{~ms}$, being very close to the $21 \mathrm{~ms}$ reported in the classic study of Rizzolatti et 
al. (1987). In the same way, the analyses revealed no difference between same and opposite hemifield for the stimuli presented inside the frame, corroborating the results of Rizzolatti et al. (1987) for conditions where the target was presented at the cued position (valid trials). The results are better explained by the premotor theory of attention and not by the shift of attention between these locations revealing the relevance of the premotor theory to the understanding of the mechanisms of visual perception. However, this finding does not exclude the hypothesis of the orientation and adjustment of the focus of attention to the shape of the rectangular area, unless the motor program activated more than one coordinate inside the frame simultaneously. Even if the motor program was prepared toward many expected locations, the area seems to be too large and had inside four possible locations where the letter could appear, with $6.4^{\circ}$ of visual angle of distance between the two more extreme locations. As the frame was presented $1.1^{\circ}$ of visual angle from fixation, the motor program would need to cover an angle of more than $153^{\circ}$ in a plane of $360^{\circ}$ for the four possible locations where the target could appear inside the frame, independent of the orientation of the frame. This area seems rather big and consuming a large amount of resources of the programming system. Another possibility would be for the motor programming to program only one location at a time. In other words, when the frame was presented the motor program might have taken as reference only the coordinates of the geometric center of the frame. But this will generate a cost to reprogram the distance among the four possible locations inside the frame. To check this possibility, an analysis was conducted for the PSS of the four locations of the letters presented inside the frame in relation to the letter presented outside and no statistically significant differences $(p>.05)$ were found among locations inside or outside. This suggests that there was a homogenous advantage for locations inside of the frame and a homogenous cost for locations outside the frame. A reasonable explanation for these findings can be that the motor program activated the map of coordinates for the orientation (left, right, below, above) where the stimuli could appear according to the frame location, but an adjustment of the resources of attention along the whole rectangular frame also occurred, giving advantage for the letter presented inside the frame independently of its location.

The comparison between the same and opposite meridian also revealed that the eccentricity of the letter presented outside the frame affected the PSS in an opposite way. This means that the letter presented more distant from the fixation (same hemifield of the frame) produced a smaller cost than the letter presented nearer (opposite hemifield). Haddad, Carreiro and Baldo (2002) showed that the distance between the fixation stimulus and the stimuli to be judged interferes in the perception of temporal order. The targets had their perceptual latency increased at peripheral locations as compared to central locations. Therefore, due to the different distances between the fixation and the letters presented inside and outside of the frame in $50 \%$ of the trials, the greater PF of the letter presented inside might not have been an effect of the different eccentricities used, but this point was better controlled in the next experiments maintaining the stimuli at the same eccentricity.

In short, the results of this experiment are similar to findings of behavioral studies which investigated the hypothesis of prior entry and are coherent with the TOJ task paradigm (Hikosaka et al., 1993; Shore et al., 2001; Stelmach \& Herdman, 1991). The results also point in the same direction as studies that support an advantage in processing for targets presented at areas delimited by cues (Castiello \& Umiltà, 1990; Galera et al., 2005; Panagopoulos et al., 2004; Posner et al., 1980) and suggest that the abrupt onset of the frame, automatically captured the resources of attention (Remington et al., 1992; Turatto et al., 2000; Yantis \& Jonides, 1990, 1996). In spite of this, we can argue that the results indicate that the borders of the frame influenced the distribution of attention, because the letters were presented close to each other with just the border between them, but nevertheless there was a perceptual facilitation for the letter presented inside. Moreover, the meridian effect found in a secondary analysis revealed that the motor activity in the programming of eye movements might have contributed to the differences in performance among meridians, while the difference between inside and outside and the PF might be better attributed to a larger concentration of attention inside of the frame.

\section{Results and Discussion of Experiment 2}

Two participants were excluded from the main analyses because PSSs were four times the standard deviation. An average of $6.4 \%$ of trials were discarded due to very long reaction times.

A repeated measures ANOVA for PSSs for each position of the first letter (inside or outside) and distance $\left(1.9^{\circ}\right.$ or $\left.5.7^{\circ}\right)$ revealed a difference between the PSS of the letter presented inside $(-38 \mathrm{~ms})$ and outside of the frame (38 ms, $F(1.14)=36.07 ; p<.0001)$. This result, as the general PF of $38 \mathrm{~ms}$, is similar to the results obtained in the first experiment and confirms the previous results. The analyses also revealed that the distance between the two letters did not produce a statistically significant difference $\left(1.9^{\circ}=-1 \mathrm{~ms}, 5.7^{\circ}=+1\right.$ $\mathrm{ms}, F(1.14)=.24 ; p=.633$ ), but there was a significant interaction between position of the first letter and distance $(F(1.14)=5.63 ; p=.033)$. The Newman-Keuls test $(p<.05)$ revealed that the PSS of condition inside at $1.9^{\circ}(-34 \mathrm{~ms})$ was not different from the PSS of condition inside at $5.7^{\circ}(-42 \mathrm{~ms})$, and there was a tendency for the condition outside the frame $\left(1.9^{\circ}=+32 \mathrm{~ms}\right.$ and $5.7^{\circ}=+44 \mathrm{~ms}, p=.06$ ). 
A) Experiment 1

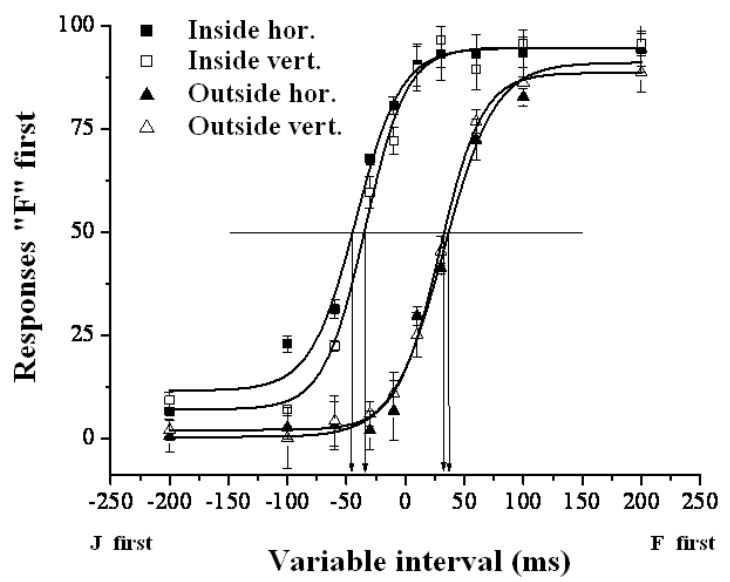

C) Experiment 3 - PSS

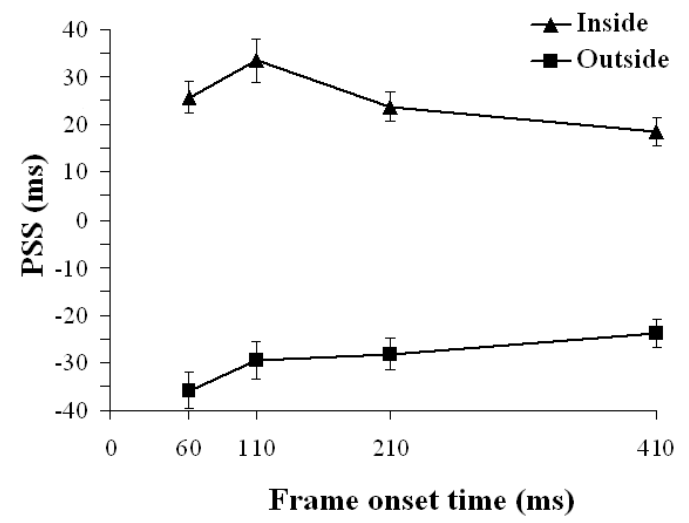

B) Experiment 2

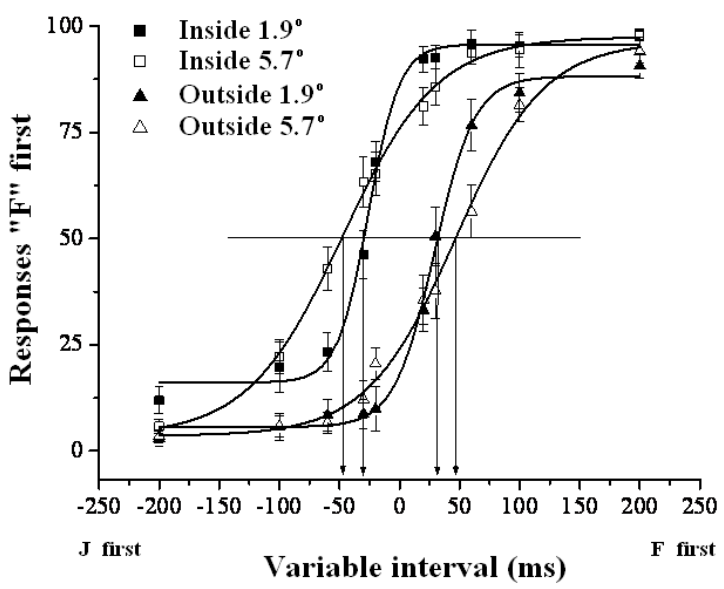

D) Experiment 3 - PF

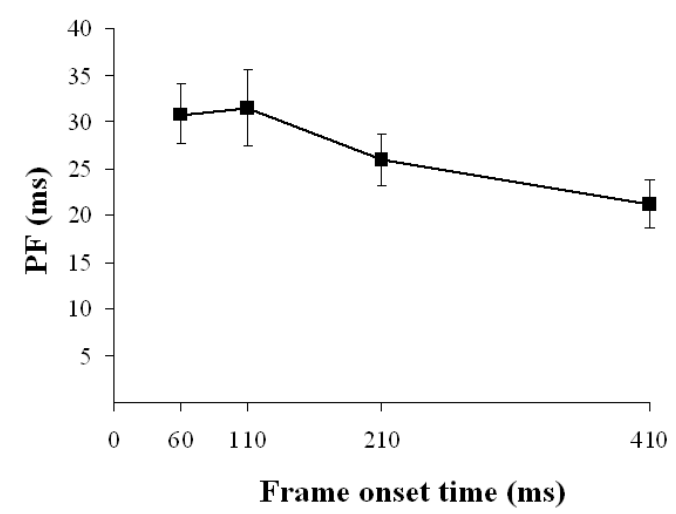

Figure 2. In Experiments 1 and 2 the fitting of the empiric values (mean of responses "F" first) through the Boltzman function for the conditions inside and outside of each frame orientation (horizontal or vertical - A) and for each distance between the letters $\left(1.9^{\circ}\right.$ and $\left.5.7^{\circ}-B\right)$ is shown in relation to the variable interval between the letters. The four arrows point to the intervals of time, corresponding to each point of subjective simultaneity (PSS) calculated for each function. In Experiment 3 the PSS of letters presented first inside or outside of the frame and the perceptual facilitation (PF), are shown as a function of the frame onset time $(60,110,210,410 \mathrm{~ms}-\mathrm{C}$ and $\mathrm{D}$, respectively). The vertical bars represent the standard error of estimate.

On the other hand, the analysis of PF and of the inclination of the function showed that the distance changed the perception of temporal order. In this experiment we analyzed the participants' judgment precision as in the study of Scharlau (2004) for each distance. The precision was obtained from the slope of the psychometric function (Sternberg \& Knoll, 1973). A steep slope indicated a large number of correct responses. The slopes and the PF obtained for each distance were submitted to independent $t$-tests $(p<.05)$. The analysis showed that the slope $(t(14)=-3.12, p=.007)$ and the PF $(t(14)=-$ $2.4, p=.03)$ were smaller when the letters were presented at $1.9^{\circ}$ (18 and $33 \mathrm{~ms}$, respectively) than at $5.7^{\circ}(32 \mathrm{~ms}$ and $43 \mathrm{~ms}$, respectively). This difference can be visualized in Figure 2B where the different slopes of the functions of the two distances show that the participants were more precise when the distance was short than when it was long. On the other hand, the PF was smaller at the short distance than when the letters were presented far apart. These results show that the short distance reduced the benefit of the letter presented inside the frame, pro-bably because at this distance the comparison between the two letters was easier. But for the long distance the opposite occurred. This suggests that the letter presented closer to the frame received a larger amount of attention than the letter presented more distant. 
These results are different from the findings of Experiment 1 and 2 of Scharlau (2004). Scharlau presented the cue and the two comparison stimuli very quickly. A gradual effect in PF in relation to the distance only occurred when the stimuli remained on the screen for a longer time, as in the present study. Scharlau (2004) argues that when the visual field is empty, it is difficult to direct attention to a determined area. However, when objects are present, attention can be more easily directed to a specific object. This idea corroborates the results found in our study and results from studies that have presented cues for long periods of time (Castiello \& Umiltà, 1990; Maringelli \& Umiltà, 1998), while no effect was found when the visual field was empty (Zimba \& Hughes, 1987).

In summary, the results suggest that the focus of attention was efficiently directed to the frame and that there was a gradual distribution of attention outside the edges of the frame. This supports the model that proposes the gradual distribution of attention through space (LaBerge \& Brown, 1989; Shulman et al., 1985). Furthermore, the PF cannot be attributed to different eccentricities between the letters, because the letters and the frame were always presented at the same distance from the fixation stimulus.

\section{Results and Discussion of Experiment 3}

An average of $5.1 \%$ of trials for each participant was discarded for the main analysis due to long reaction times and one participant was excluded because PSSs were four times the standard deviation.

A repeated measures ANOVA for PSSs for each position of the first letter (inside or outside) and the frame onset time $(60,110,210$ or $410 \mathrm{~ms})$, showed that the PSS of letter presented inside $(-29 \mathrm{~ms})$ was statistically different of PSS of the letter presented outside $(+25 \mathrm{~ms}, F(1.13)=$ $126.82 ; p<.0001)$. The general PF, estimated through the PSSs of conditions inside and outside, revealed an advantage of $27 \mathrm{~ms}$ for information presented inside of the frame. The frame onset time produced a significant effect on the PSS $(F(3.39)=4.22 ; p<.011)$ and there was an interaction between position and frame onset time $(F(3.39)=4.02 ; p<.014)$. A Newman - Keuls post hoc test $(p<.05)$ showed that the average of the PSS of the frame onset time of $60 \mathrm{~ms}(-5 \mathrm{~ms})$ was different from PSS of frame onset time of $110 \mathrm{~ms}(+2 \mathrm{~ms})$ and this last one was different from PSS of frame onset time of 210 and $410 \mathrm{~ms}$ ( -2 and $-3 \mathrm{~ms}$, respectively). Other paired comparisons (Newman - Keuls post hoc test) for the two factors revealed that the gain for the inside position for frame onset time of $60 \mathrm{~ms}$ and $410 \mathrm{~ms}(-36$ and $-24 \mathrm{~ms}$, respectively) was near statistical significance $(p=0.07)$. When the letter was presented outside, there was a difference between the frame onset times of $110 \mathrm{~ms}$ and $410 \mathrm{~ms}$ ( $+33 \mathrm{~ms}$ and $+19 \mathrm{~ms}$, Figure $2 \mathrm{C})$. This difference between the PSSs in relation to the frame onset time produced a peak in PF at $110 \mathrm{~ms}$ of cue presentation, decreasing gradually until it achieved smaller values at
$410 \mathrm{~ms}$. A repeated measures ANOVA for PF revealed a main effect for the four $\mathrm{PF}$ of each frame lead time $(F(3.39)=4,02 ; p<.014)$. The Newman - Keuls post hoc test showed a large PF for short intervals of 60 and $110 \mathrm{~ms}$ (31 ms and 32, respectively) and smaller PF for the longest interval of $410 \mathrm{~ms}(21 \mathrm{~ms})$.

This pattern of results with a peak of activation at 110 $\mathrm{ms}$ and a decay of the PF thereafter can be visualized in Figure 2D and it is in agreement with studies that have investigated the temporal dynamics of visual attention in RT tasks (Cheal \& Lyon, 1991; H. J. Müller \& Rabbitt, 1989; Nakayama \& Mackeben, 1989; Posner \& Cohen, 1984). These studies propose that abrupt onsets presented in the periphery of the visual field capture attention automatically when short intervals (until $100 \mathrm{~ms}$ ) are used between these stimuli and targets. Thereby, the results of this experiment seem to show an automatic distribution of visual attention to the area delimited by a rectangular frame. In addition, the pattern of results found here with these cue lead times does not seem to point to an inhibition of the information presented inside the frame, but instead a decrease in advantage seems more in accordance with the idea of different phases of allocation of attention with an automatic transitory initial phase, while later it may be followed by a voluntary component that can be extended for a longer period (Cheal \& Lyon, 1991; H. J. Müller \& Rabbitt, 1989; Nakayama \& Mackeben, 1989).

In summary, the main contributions of this study are the results that support the idea of a more flexible attentional focus, able to be automatically adjusted to a rectangular shape and with edges that seem to respect the limits of the shape of the stimulus used as cue, but with a gradual decrease in processing efficiency beyond its borders.

\section{General Discussion}

In this study we investigated the distribution of visual attention and the possibility of its resources to be more concentrated inside than outside of an abrupt onset frame. The present experiments were based on evidence that abrupt onsets capture attention automatically (Remington et al., 1992; Turatto et al., 2000; Yantis \& Jonides, 1990, 1996), as well as on evidence that geometric figures with specific shapes can modulate the distribution of visual attention (Benso et al., 1998; Egly \& Homa, 1984; Galera et al., 2005; Juola et al., 1991; M. M. Müller \& Hübner, 2002; Panagopoulos et al., 2004). Moreover, according to the prior entry hypothesis, the displacement of visual attention for a specific stimulus should influence the strength (McDonald et al., 2005) or the speed (Vibell et al., 2007) of processing of the attended information, shortening the perceptual latency of an attended stimulus (Shore et al., 2001; Stelmach \& Herdman, 1991; Sternberg $\&$ Knoll, 1973). Thus, the initial hypothesis was that the abrupt onset of a figure with delimited borders should initiate the automatic orientation of visual attention chan- 
ging the perception of temporal order of the signals, revealing the prior entry effect. Therefore, in three experiments, two letters were presented in sequence with a variable interval between them and one letter inside and the other outside an abrupt onset frame. Participants performed a TOJ task of which letter was presented first, giving a relative estimate of the advantage of information presented inside in relation to information presented outside of the frame, or the perceptual facilitation, being similar to studies that compared situations where the target was presented inside a peripheral cue (valid trials) and outside this cue (invalid trials).

The results of the three experiments showed that the presentation of one letter inside and the other outside the frame changed the perception of temporal order. An advantage in all experimental conditions occurred for the letter presented inside of the frame in relation to the letter presented outside. These results are in accordance with studies that have been investigating the effect of prior entry and suggest that the effect can be attributed to greater concentration of resources of attention inside the frame.

Results of Experiment 1 showed greater PF when the frame was presented in the horizontal rather than in the vertical orientation (this analysis was not possible in Experiment 3 due to a very small number of trials that would be available for analysis, considering the five frame lead times). Similar results of RT tasks suggest that the larger advantage found for the horizontal meridian can be the product of a greater distribution of attention along this meridian (Altpeter et al., 2000; Galera et al., 2005; Panagopoulos et al., 2004). But different results for a comparison of the horizontal and vertical meridians were also found by Carrasco et al. (2001) who reported no difference between conditions where attention was displaced and those where it was not displaced along different meridians. Thus, the results presented here should be analyzed carefully, because visual constraints such as different concentrations of cells in the different visual fields can produce such effects (Carrasco et al., 2001; Curcio \& Allen, 1990). This difference in performance needs to be further investigated in other experiments to determine if the effect is caused by unequal distribution of attention on each meridian or by visual constraints.

In Experiment 2, the manipulation of the distance between the letter presented inside and the letter presented outside of the frame demonstrated that the accuracy was greater when the letter presented outside was closer to the frame than when it was farther away from it. Again, these results replicated studies that have demonstrated an automatic allocation of attention to cues presented in the periphery of the visual field and showed a decrease in performance with increasing distance from the cue, which suggests a gradual distribution of attention to stimuli presented outside of the point initially attended (LaBerge \& Brown, 1989; Shulman et al., 1985). These results indicate that the borders of the focus of attention might not have been precisely delimited by the form of the object, but faded gradually (Castiello \& Umiltà, 1990). Although this might have happened, recent neurophysiological and behavioral evidence suggests that an area of suppression around the attended area can exist together with the gradual decrease of attention outside the limits of the cue (N. G. Müller \& Kleinschmidt, 2004; N. G. Müller, Mollenhauer, Rösler, \& Kleinschmidt, 2005). This leaves open the possibility of a flexible focus, able to be adjusted to the shape and size of the object with a gradual decrease outside this area. In other words, the areas of suppression seem to be well delimited and very close to the borders of the focus. If the focus is adjusted to the size and shape of an object, these areas could be defined by the characteristics of the attended object. Stimuli that would fall inside these areas of suppression should have their processing suppressed, but at farther away areas a gradual decrease of attention should occur. Thus, the results of Experiment 2 are still in accordance with the idea of a flexible focus of attention. Furthermore, the PF found in Experiment 2 was very similar to the PF found in Experiment 1, suggesting that the latter effect cannot be attributed primarily to the different eccentricities of the stimuli used.

Experiment 3, as well as Experiment 2, looked for additional evidence that the change in the perception of temporal order was induced by the different distribution of visual attention. We manipulated the interval of presentation of the frame to verify the temporal dynamics of attention when a TOJ task was used. The results showed that the judgment of temporal order was influenced by the interval between the presentation of the frame and the presentation of the first target, which varied between 60 and $410 \mathrm{~ms}$. The magnitude of the advantage produced by the frame reached a peak at $110 \mathrm{~ms}$. This advantage decreased when the first target was presented after longer intervals. The results follow the same patterns of PF found in different studies that examined the temporal dynamics of visual attention indicating that the abrupt onset of the frame initiated an automatic displacement of attention to its area (Cheal \& Lyon, 1991; H. J. Müller \& Rabbitt, 1989; Nakayama \& Mackeben, 1989). The decrease in PF with the further increase of the interval suggests that after the initial automatic activation of the resources of attention, the concentration of resources inside the frame can decrease as a result of the change of the visual response to a voluntary control of the distribution of attention. But, further evidence is needed, because this pattern of results could also have a contribution by sensory factors (Schneider \& Bavelier, 2003). Interactions between the characteristics of stimuli presented with intervals of time and space that are very small might interfere with the results independent of the displacement of visual attention to the cued location.

Finally, the results of this study provide evidence for the automatic distribution of visual attention, using a 
measure which estimated the temporal advantage of stimuli presented inside of a frame. The results are best explained by models that propose a spatial distribution of visual attention (Eriksen \& St. James, 1986; LaBerge, 1983; LaBerge \& Brown, 1989; Posner et al., 1980), and are coherent with the idea of a flexible focus of attention which can be adjusted to the size and shape of objects presented in the visual field (Galera et al., 2005; Panagopoulos et al., 2004). On the other hand, we cannot discard the possibility of mechanisms of object-based selection that might have contributed to the advantage of attention on PF found in the present experiments. This hypothesis would be related to the proposal of modulation of sensory and spatial representations early in the visual system by attention which would select the location covered by the object (N. G. Müller \& Kleinschmidt, 2003), with the automatic distribution or "spread" of attention inside the area delimited by the borders of the attended object (Abrams \& Law, 2000; Brown, Breitmeyer, Leighty, \& Denney, 2006; Egly, Driver, \& Rafal, 1994; N. G. Müller \& Kleinschmidt, 2003). This approach has been successful, showing that both mechanisms of spatialbased and object-based selection can exist and contribute to the processing advantage related to visual attention. Studies that manipulate the size and the shape of the object, together with the manipulation of the distance of the stimuli relevant to the task from the borders of the object, could provide new information about the mechanisms of the distribution of visual attention between and beyond objects and spatial locations.

\section{References}

Abrams, R. A., \& Law, M. B. (2000). Object-based visual attention with endogenous orienting. Perception \& Psychophysics, 62, 818-833.

Altpeter, E., Mackeben, M., \& Treuzettel-Klosinski, S. (2000). The importance of sustained attention for patients with maculopathies. Vision Research, 40, 1539-1547.

Benso, F., Turatto, M., Mascetti, G. G., \& Umiltà, C. (1998). The time course of attention focusing. European Journal of Cognitive Psychology, 10, 373-388.

Brown, J. M., Breitmeyer, B. G., Leighty, K. A., \& Denney, H. I. (2006). The path of visual attention. Acta Psychologica, 121, 199-209.

Carrasco, M., Talgar, C. P., \& Cameron E. L. (2001). Characterizing visual performance fields: Effects of transient covert attention, spatial frequency, eccentricity, task and set size. Spatial Vision, 15, 61-75.

Castiello, U., \& Umiltà, C. (1990). Size of the attentional focus and efficiency of processing. Acta Psychologica, 73, 195209.

Cheal, M., \& Lyon, D. R. (1991). Central and peripheral precuing of forced-choice discrimination. Quarterly Journal of Experimental Psychology, 43, 859-880.

Cole, G., Gellatly, A., \& Blurton, A. (2001). Effect of object on the distribution of visual attention. Journal of Experimental Psychology: Human Perception \& Performance, 27, 13561368.
Curcio, C. A., \& Allen, K. A. (1990). Topography of ganglion cells in human retina. Journal of Comparative Neurology, 300, 5-25.

Craighero, L., \& Rizzolatti, G. (2005). The premotor theory of attention. In L. Itti, G. Rees, \& J. Tsotsos (Eds.), Neurobiology of attention (pp. 181-186). San Diego, CA: Elsevier Academic Press.

Egly, R., Driver, J., \& Rafal, R. D. (1994). Shifting visual attention between objects and locations: Evidence from normal and parietal lesion subjects. Journal of Experimental Psychology: General, 123, 161-177.

Egly, R., \& Homa, D. (1984). Sensitization of the visual field. Journal of Experimental Psychology: Human Perception \& Performance, 10, 778-793.

Eimer, M. (2000). An ERP study of sustained spatial attention to stimulus eccentricity. Biological Psychology, 52, 205-220.

Eriksen, C. W., \& St. James, J. D. (1986). Visual attention within and around the field of focal attention: A zoom lens model. Perception \& Psychophysics, 40, 225-240.

Eriksen, C. W., \& Yeh, Y. Y. (1985). Allocation of attention in the visual field. Journal of Experimental Psychology: Human Perception and Performance, 11, 583-597.

Galera, C., von Grünau, M., \& Panagopoulos, A. (2005). Automatic focusing of attention on object size and shape. Psicológica, 26, 147-160.

Haddad, H., Jr., Carreiro, L. R. R., \& Baldo, M. V. C. (2002). Modulation of the perception of temporal order by attentional and pré-attentional factors. Brazilian Journal of Medical and Biological Research, 35, 979-983.

Hikosaka, O., Miyauchi, S., \& Shimojo, S. (1993). Focal visual attention produces illusory temporal order and motion sensation. Vision Research, 33, 1219-1240.

Juola, J. F., Bouwhuis, D. G., Cooper, E. E., \& Warner, C. B. (1991). Control of attention around the fovea. Journal of Experimental Psychology: Human Perception \& Performance, 17, 125-141.

Juola, J. F., Crouch, T., \& Cocklin, T. (1987). Voluntary control of attention near the fovea. Acta Psychologica, 63, 207-217.

Laberge, D. (1983). Spatial extent of attention to letters and words. Journal of Experimental Psychology: Human Perception \& Performance, 9, 371-379.

Laberge, D., \& Brown, V. (1989). Theory of attentional operation in shape identification. Psychological Review, 96, 101-124

McDonald, J. J., Teder-Sälejärvi, W. A., Di Russo, F., \& Hillyard, S. A. (2005). Neural basis of auditory-induced shifts in visual time-order perception. Nature Neuroscience, 8,1197 1202.

Maringelli, F., \& Umiltà, C. (1998). The control of the attentional focus. European Journal of Cognitive Psychology, 10, 225-246.

Müller, H. J., \& Rabbitt, P. M. A. (1989). Reflexive and voluntary orienting of visual attention: Time course of activation and resistance to interruption. Journal of Experimental Psychology: Human Perception \& Performance, 15, 315-330.

Müller, M. M., \& Hübner, R. (2002). Can the spotlight of attention be shaped like a doughnut? Psychological Science, 13, 119-124.

Müller, N. G., \& Kleinschmidt, A. (2003). Dynamic interaction of object- and space-based attention in retinotopic visual areas. The Journal of Neuroscience, 23, 9812-9816. 
Müller, N. G., \& Kleinschmidt, A. (2004). The attentional 'spotlight's' penumbra: Center surround modulation in striate cortex. Neuroreport, 15, 977-980.

Müller, N. G., Mollenhauer, M., Rösler, A., \& Kleinschmidt, A. (2005). The attentional field has a Mexican hat distribution. Vision Research, 45, 1129-1137.

Nakayama, K., \& Mackeben, M. (1989). Sustained and transient components of focal visual attention. Vision Research, 29, 1631-1647.

Panagopoulos, A., von Grünau, M. W., \& Galera, C. (2004). Attentive mechanisms in visual search. Spatial Vision, 17, 353-371.

Posner, M. I., \& Cohen, Y. (1984). Components of visual orienting. In H. Bouma \& G. G. Bouwhuis (Eds.), Attention and performance $X$ (pp. 531-556). Hillsdale, NJ: Erlbaun.

Posner, M. I., Snyder, C. R. R., \& Davidson, B. J. (1980). Attention and the detection of signals. Journal of Experimental Psychology: General, 109, 160-174.

Remington, R. W., Johnston, J. C., \& Yantis, S. (1992). Involuntary attention capture by abrupt onsets. Perception \& Psychophysics, 51, 279-290.

Rizzolatti, G., Riggio, L., Dascola, I., \& Umiltà, C. (1987). Reorienting attention across the horizontal and vertical meridians: Evidence in favor of a premotor theory of attention. Neuropsychologia, 25, 31-40.

Scharlau, I. (2004). The spatial distribution of attention in perceptual latency priming. The Quarterly Journal of Experimental Psychology, 57, 1411-1436.

Schneider, K. A., \& Bavelier, D. (2003). Components of visual prior entry. Cognitive Psychology, 47, 333-366.

Shore, D. I., Spence, C., \& Klein, R. M. (2001). Visual prior entry. Psychological Science, 12, 205-212.

Shulman, G. L., Wilson, J., \& Sheehy, J. B. (1985). Spatial determinants of the distribution of attention. Perception \& Psychophysics, 37, 59-65.

Stelmach, L. B., \& Herdman, C. M. (1991). Directed attention and perception of temporal order. Journal of Experimental Psychology: Human Perception and Performance, 17, 539550.

Sternberg, S., \& Knoll, R. L. (1973). The perception of temporal order: Fundamental issues and a general model. In S. Kornblum (Ed.), Attention and performance IV (pp. 629-685). New York: Academic Press.

Turatto, M., Benso, F., Facoetti, A., Galfano, G., Mascetti, G. G., \& Umiltà, C. (2000). Automatic and voluntary focusing of attention. Perception and Psychophysics, 62, 935-952.

Vibell, J., Klinge, C., Zampini, M., Spence, C., \& Nobre, A. C. (2007). Temporal order is coded temporally in the brain: Early event-related potential latency shifts underlying prior entry in a cross-modal temporal order judgment task. Journal of Cognitive Neuroscience, 19, 109-120.

Yantis, S., \& Jonides, J. (1990). Abrupt visual onsets and selective attention: Voluntary versus automatic allocation. Journal of Experimental Psychology: Human Perception \& Performance, 16, 121-134.

Yantis, S., \& Jonides, J. (1996). Attentional capture by abrupt onsets: New perceptual objects or visual masking? Journal of Experimental Psychology: Human Perception \& Performance, 22, 1505-1513.

Zimba, L. D., \& Hughes, H.C. (1987). Distractor-target interactions during directed visual attention. Spatial Vision, 2, 117-149. 